\title{
Nitrogen-fixation by Mixed Cultures of Aerobic and Anaerobic Micro-organisms in an Aerobic Environment
}

\author{
By M. A. LINE AND MARGARET W. LOUTIT \\ Department of Microbiology, University of Otago, Dunedin, New Zealand
}

(Received 23 May 1972; revised 22 August 1972)

In recent years the ability of a variety of putative nitrogen-fixing bacteria or yeasts to fix nitrogen has been disputed (Parejko \& Wilson, I968; Hill \& Postgate, I969; Millbank, I $969 ; 1970$ ). This communication discusses the failure of oligonitrophilic organisms from local soils and from overseas workers to fix nitrogen, and provides a new explanation of apparent nitrogen-fixation by these organisms.

In a recent study of soil samples from a number of New Zealand localities and from an earlier study of a single tussock-grassland locality (Line \& Loutit, I97I) non-nitrogenfixing oligonitrophilic organisms were present in considerable numbers in these soils. Isolates belonging to this group were identified as species of Pseudomonas, Bacillus, Enterobacter and yeast, although true nitrogen-fixing strains of Bacillus and Enterobacter were also found. In view of the absence of nitrogen-fixation by species of Pseudomonas obtained from New Zealand soils, putative nitrogen-fixing strains of this genus were obtained from overseas workers and tested for acetylene-reduction and nitrogen uptake as described previously (Line \& Loutit, I97I). Strains tested included II isolates from the Meiklejohn \& Weir (1968) collection and isolates from Dr J. P. Voets and Dr E. A. Paul.

In no case was acetylene-reduction detected for any isolate following aerobic incubation on unsupplemented or soil-extract supplemented nitrogen-deficient agar slopes. Similarly no significant nitrogen uptake was observed in aerobically incubated liquid cultures following incubation at $28^{\circ} \mathrm{C}$ for I month. In contrast, cultures of Azotobacter and Beijerinckia strains grown under the same conditions showed strongly positive acetylene-reduction and fixed 49 and $95 \mu \mathrm{g} \mathrm{N} / \mathrm{ml}$ liquid medium respectively.

A puzzling feature of the investigation of isolates obtained from New Zealand soils was the occasional acetylene-reduction by crude liquid cultures of less than $\mathrm{I} \mathrm{cm}$ depth. This result, however, was not repeatable following aerobic purification of the organisms. Although acetylene-reduction was demonstrable both in the original enrichments and following one aerobic subculture on to nitrogen-deficient agar medium, single colonies on the latter medium were uniformly incapable of acetylene-reduction, with this capacity only observable on testing cells from the confluent region of growth.

The problem was resolved when the enrichment cultures were subcultured and incubated anaerobically. Under these conditions a nitrogen-fixing Clostridium strain became apparent, this being incapable of aerobic growth in pure culture. It therefore appeared that sufficiently reduced conditions were produced in the liquid enrichments by non-nitrogen-fixing organisms to allow the development of the clostridia, and on subsequent testing on agar slopes in sealed containers the aerobic organisms again produced the necessary reduced conditions for growth and acetylene-reduction by the clostridia.

As a consequence of these results, a strain of Clostridium butyricum isolated from soil was tested for 'aerobic' acetylene-reduction on agar slopes in association with a variety of 
oligonitrophilic species. Positive results were obtained in all cases, although the different associations produced widely varying levels of acetylene-reduction. Of note was a very marked acetylene-reduction response resulting from a mixture of Clostridium and Pseudomonas azotogensis provided by Dr Voets. The levels of reduction shown by this association following aerobic incubation were considerably higher than those obtained using any other combination of aerobe plus Clostridium.

From the studies carried out it has been shown that a mixed culture of nitrogen-fixing Clostridium and an aerobic organism are capable of nitrogen-fixation in a seemingly aerobic environment. In future cases of apparent aerobic nitrogen-fixation by a previously unreported species, a check for Clostridium by anaerobic incubation may be a worth-while precaution.

We are grateful to Dr J. Antheunisse, Dr E. A. Paul and Dr J. P. Voets for kindly providing cultures. M. A. L. is a Miss E. L. Hellaby Indigenous Grasslands Research Fellow.

\section{REFERENCES}

Hill, S. \& Postgate, J. R. (1969). Failure of putative nitrogen-fixing bacteria to fix nitrogen. Journal of General Microbiology 58, 277-285.

LiNe, M. A. \& LoutrT, M. W. (197I). Non-symbiotic nitrogen-fixing organisms from some New Zealand tussock grassland soils. Journal of General Microbiology 66, 309-318.

MeiklejoHN, J. \& WeIR, J. B. (1968). Nitrogen-fixers - pseudomonads and other aerobic bacteria - from Rhodesian soils. Journal of General Microbiology 5o, 487-496.

Millbank, J. W. (I969). Nitrogen fixation in moulds and yeasts - a reappraisal. Archiv für Mikrobiologie 68, 32-39.

MillbaNk, J. W. (1970). The effect of conditions of low oxygen tension on the assay of nitrogenase in moulds and yeasts using the acetylene reduction technique. Archiv für Mikrobiologie 72, 375-377.

Parejko, R. A. \& Wilson, P. W. (1968). Taxonomy of Azotomonas species. Journal of Bacteriology 95, I43-I 46 . 